\title{
Does a critical speed for railroad vehicles exist?
}

True, Hans

Published in:

Proceedings of the ASME/IEEE Joint Railroad Conference

Link to article, DOI:

10.1109/RRCON.1994.289013

Publication date:

1994

Document Version

Publisher's PDF, also known as Version of record

Link back to DTU Orbit

Citation (APA):

True, H. (1994). Does a critical speed for railroad vehicles exist? In Proceedings of the ASME/IEEE Joint Railroad Conference (pp. 125-131). IEEE. https://doi.org/10.1109/RRCON.1994.289013

\section{General rights}

Copyright and moral rights for the publications made accessible in the public portal are retained by the authors and/or other copyright owners and it is a condition of accessing publications that users recognise and abide by the legal requirements associated with these rights.

- Users may download and print one copy of any publication from the public portal for the purpose of private study or research.

- You may not further distribute the material or use it for any profit-making activity or commercial gain

- You may freely distribute the URL identifying the publication in the public portal

If you believe that this document breaches copyright please contact us providing details, and we will remove access to the work immediately and investigate your claim. 


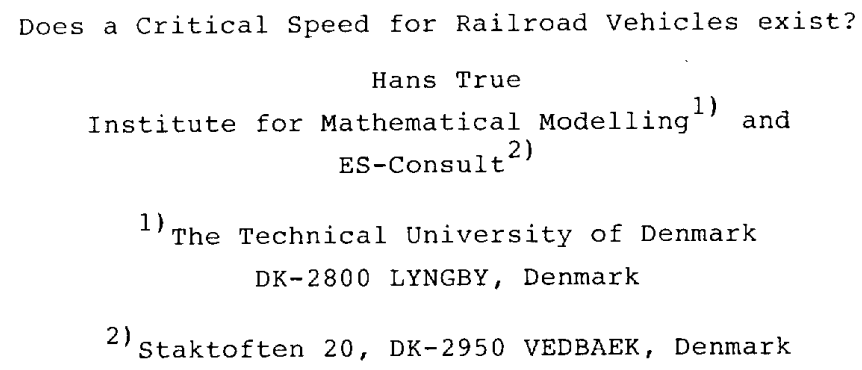

In this article we discuss the definition and existence of a critical speed for the onset of hunting of railroad vehicles. First the field test situation is described. It is argued that the important problem is the determination of the forces and accelerations in the vehicle and the rails, which may be large even when the vehicle does not hunt. Next we discuss the relevance of the critical speed in railway engineering. We conclude that it is desirable to know the speed below which a vehicle will not hunt, since hunting always is connected with large wheel-rail forces. Next the modelling of railroad vehicles is discussed and the nonlinear aspects are emphasized. Next we explain why it is necessary to reformulate the mathematical problem for calculation of the critical speed due to the nonlinear character of the modelling. It is suggested that another critical speed be defined for a railroad vehicle. It can be calculated without great effort using a well functioning simulation program, and we explain how to determine the "new" critical speed theoretically. It yields a "global" criterion for the onset of hunting, in contrast to the linear stability criterion, which is still commonly used. The linear eigenvalue problem yields a bifurcation point, which in general will not be the lowest parameter value for existence of oscillating solutions in a nonlinear dynamical system.

We suggest that the railroad industry abandon the concept of "linearized critical speed", since it is generally irrelevant for the onset of oscillations in nonlinear dynamics. Instead we suggest the use of a "nonlinear critical speed" as an acceptance criterion for railroad vehicles.

\section{THE FIELD TEST SITUATION}

The auto oscillation of railroad vehicles at speed - called hunting - is a well known phenomenon which was first described by George Stephenson (1821). When a railroad vehicle accelerates, hunting starts in a very narrow speed interval. A plot of the lateral acceleration measured at an axle box looks characteristically like the plot shown on Figure 1.

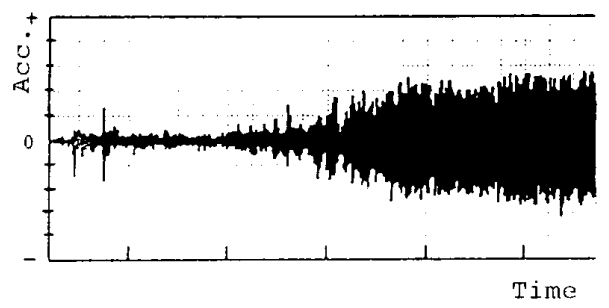

Figure 1. Time series of the lateral acceleration of the bogie frame showing the start of hunting. Each unit on the vertical axis corresponds to $0.5 \mathrm{~m} / \mathrm{s}^{2}$. 
The jump in amplitude of the oscillation of the lateral acceleration is so sudden that it easily can be related to the speed of the vehicle at that instant within say $3 \mathrm{~km} / \mathrm{h}$. It is therefore tempting to define a speed in the "center" of that speed range as a "critical speed". The problem is, however, that this "critical speed" is not easily reproducible. Therefore we have shown no units on the abscissa of Figure 1.

It is well known that the so called critical speed depends on many external parameters such as the weight of the vehicle and the weight distribution, the magnitudes of various moments of inertia, the adhesion between wheel and rail, the curvature of the track, suspension characteristics, the shape of the vehicle and its running gear and very importantly-all parameters that combine into the quantity wheel-rail-contact geometry.

For a specific vehicle many of these parameters are given and fixed, and we are thus left with the indeterminable parameters relating to the track. The coefficient of adhesion can be measured, and in certain geographical areas with constant weather conditions it will change very little within that area. But even then - with a well defined wheel profile and a first class track - the so called critical speed in most cases will vary from line to line. Furthermore, if the test is reversed, in the sense that we measure the speed at which the hunting stops when the vehicle slows down (without applying the brakes), then it is most often found that the hunting stops at a LOWER SPEED than the one where hunting starts during acceleration. In some cases there is a considerable difference between the two "critical speeds". Wear of wheels also influences the "critical speed", so it changes with time.

We are thus led to the conclusion that a unique "critical speed" for a vehicle, above which hunting starts and below which hunting stops, DOES NOT EXIST IN REALITY. This conclusion holds true even when the coefficient of adhesion and the track geometry is known with the highest achievable accuracy.
IS IT NECESSARY TO KNOW A CRITICAL SPEED?

Hunting creates large forces between the wheel and the rail which affect the track geometry adversely. In passenger vehicles the comfort suffers, and the costs of maintenance for both rolling stock and track increase. Hunting, therefore, is certainly undesirable, but other circumstances also create wheel-rail forces, which may be as large as the forces caused by hunting. These forces show up in acceleration and track force measurements, and why should the railroad companies then bother about hunting in particular?

Today high speed trains are optimized, also with respect to the track forces (with or without hunting) for the lines they run on. The existing, or planned, track geometry is the basis for a design optimization largely based on numerical simulations of the vehicle dynamics and wheelset wear. This technique works well, as long as these trains are confined to a network consisting of a few lines, but it is virtually impossible to do such an optimization for a freight car, which can move all over North America. We can take wheel wear into consideration in the optimization, but the track is also worn, and how should that be included in the optimization, since that wear depends on the entire amount of traffic running over the lines and not only on the optimized vehicles.

Although the railroad company usually pays for the maintenance of both vehicles and tracks, the track department must pay for the extra maintenance caused by dynamically bad vehicles, and the department of rolling stock must pay for the additional maintenance of vehicles running on bad track. This traditional conflict makes it meaningful to define standards for both track and rolling stock, which, although they relate to only one of the two groups, are based on reasonable que 1:- fications defined by the other group. As an example, take the standards for track that must carry 35 tonnes axle load set by the vehicle designers - and finance group.

Since hunting is hard on tracks as woll as vehicles, it is clearly desirable to avoia 
hunting altogether on all lines and at all speeds for which a vehicle is allowed. Therefore it is also desirable to determine a critical speed for a vehicle - but how? Can the theoretical investigations of railroad vehicle models be of any help?

\section{MATHEMATICAL MODEZLING OF VEHICLE DYNAMICS}

A railway vehicle is considered as a multibody in mechanics. The number of degrees of freedom combined with the nonlinearities in the problem forces the investigator of the dynamics of railroad vehicles to use numerical methods. Today there exist several codes for the modelling of railroad vehicles, and some of them are combined with solvers of the resulting system of nonlinear ordinary differential equations so simulations of the dynamics of a vehicle can be performed. The great advantage of this method is that the experimental situation is well defined and controllable. The nonlinearities in the model - most notably the creepage-creep force modelling - constitute a great challenge to the modeller, but today good models and proven approximative methods exist and are applied. Their results have often been verified by experiments and field tests. We may therefore conclude, that it is possible today to construct a satisfactory model of a moving railway vehicle including the nonlinear mechanics.

When we want to investigate the nonlinear dynamics of a nonlinear mechanical system it is a necessary condition for a good result that the nonlinear mechanics is correctly modelled, or at least well approximated, but it is by no means a sufficient condition. A good modelling can not alone guarantee good results of a dynamical investigation. The mathematics must be correct too. Nonlinear mechanics is not the same as nonlinear dynamics. Nonlinear mechanics is the formulation of the nonlinearities in the system as mathematical expressions; it is a problem of physics. Nonlinear dynamics is a set of mathematical laws for the systems of equations, which comprise the mathematical model. The mathematical theory for nonlinear systems differs strongly from the much simpler linear theory, with which all engineering students become familiar. There exist concepts in nonlinear mathematics which do not exist or have any parallel in linear mathematics. Lack of uniqueness even locally - is a distinguishing feature of most nonlinear problems and chaos only exists in nonlinear problems - only to mention two important examples. In parameter dependent nonlinear problems a concept like "bifurcation" plays an important role in the description of the overall behaviour of a dynamical system. In a railroad context this was first mentioned by Huilgol (1978) and Moelle and Gasch (1982). The concept has no parallel in linear theory.

An autonomous parameter dependent nonlinear system can be characterized in a certain parameter range by its "attractors". This is synonymous with asymptotically stable solutions. For any initial condition the evolution of a dynamical system will either lead to a blow up or end on an attractor - the mathematical theory says "asymptotically" as time approaches infinity, but in reality the solution will be "sufficiently close" to the attractor after a finite time. The attractor may be an equilibrium point representing a steady state or a limit cycle representing a solution periodic in time, but other possibilities also exist. The time evolution from the initial conditions to the attractor is called the transient. In a non-autonomous nonlinear system with given time dependent forcing, the solution may never settle to an attractor, and the entire solution as a function of time is thus a transient. This is very often the case in realistic numerical simulations of the dynamics of a vehicle on a given railroad line, where the measured track geometry is used as an input, which is transformed into a time dependent forcing of the system using the speed of the vehicle. Such transients are often complicated functions of time and they are often chaotic with surprisingly large amplitudes, True and Jensen (1993c). Unfortunately very little is known about chaotic transients today, but the chaotic nature of a transient can be the 
cause of certain unexplained discrepancies between field test results and numerical simulations of the dynamics of a railroad vehicle.

A successful numerical simulation of the motion of a railroad vehicle will, of course, look very much like the measured results from field tests (see Figure 1), and they suffer from the same deficiences when it comes to use them to determine a critical speed for a given vehicle. We need to eliminate the influence of the disturbances from the model of the real track. This can only be done in a numerical experiment. The elimination of the disturbances will force the solution of a numerical experiment to approach an attractor. We can thus concentrate our efforts on the much simpler mathematical problem of finding the attractors (asymptotically stable solutions) and characterizing them in dependence on the speed as the control parameter. By varying the coefficient of adhesion, the gauge and other relevant wheel-rail contact parameters, we can then find the minimal speed for which a time periodic asymptotically stable solution exists.

This sounds very familiar, but it is very important that the reader will learn and acknowledge that a classical stability analysis will not in general yield a critical speed for the nonlinear system. This is one of the points where the mathematical results of nonlinear dynamics differ from those of linear dynamics.

\section{CALCULATION OF THE CRITICAL SPEED}

Consider the steady solution of our nonlinear dynamical problem. It exists for all values of of the speed, $V$, and it is asymptotically stable for all positive $V$ smaller than $V_{0}$. In order to find the critical speed in our dynamical problem, the correct formulation of the related mathematical problem is:

Find the minimal speed $v_{C}$ at which any other stable solution exists, when all relevant parameters are varied over their respective ranges.
Remarks :

1. When a nonlinear problem possesses more than one asymptotically stable solution, then each of these solutions is connected to a set of initial values - the so called "basin of attraction". All transients from a basin of attraction will tend towards the corresponding stable solution as $t \rightarrow \infty$. With the new definition of the critical speed we guarantee, that all physically reasonable transients in our vehicle dynamical problem will tend towards the steady solution when $\mathrm{V}<\mathrm{V}_{\mathrm{C}}$. Theoretically some transients may tend to infinity (blow up), but this is not new. Therefore hunting is theoretically impossible for $V<V_{C}$.

2. In some cases $V_{C}=V_{O}$, which is the linear critical speed. Since in general $\mathrm{v}_{\mathrm{c}}<\mathrm{v}_{\mathrm{o}}$ it is wrong to denote $v_{o}$ the critical speed.

Mathematicians and physicists have for a long time presented the qualitative behavior of nonlinear dynamical problems on so called bifurcation diagrams. They yield a good survey of the number of solutions in dependence on one or more control parameters. In railroad vehicle dynamics bifurcation diagrams were used by Moelle and Gasch (1982), Gasch et al. (1984), Jaschinski (1990), Knudsen et al. (1991) and the author, True and Kaas-Petersen (1984), Kaas-Petersen and True (1986), True (1988), True and Jensen (1993c) and in a survey True (1993a). In all these cases $V_{C}<V_{0}$. A case where $V_{C}=V_{0}$ is described in another survey, True (1993b).

\section{CALCULATION OF THE CRITICAL SPEED CONTINUED}

When we only want to find the critical speed, $V_{C}$, it is not necessary to analyze the dynamics of the nonlinear system for all speeds. The following method will lead us to the goal.

1. Find the time periodic oscillation to which the steady motion loses its stability. 2. Take this time periodic solution as the initial condition and integrate the nonlinear system when $V$ decreases slowly. At a certain 
value of $V$ the oscillation stops. Determine the value.

3. Vary the other parameters over their respective ranges and repeat the second step for each combination. Thereby, a set of values $V$ is created. The smallest value $V_{c}$ in the set is the critical speed we are looking for.

1 additional comments. The easiest way to find the periodic motion numerically is to start a time integration at a parameter value $\mathrm{V}$ above $v_{0}$ with all state variables equal to zero in the initial condition. Since the steady solution is unstable for $V>V_{0}$, the transient solution found by the integration will tend to the asymptotically stable periodic solution.

2 additional comments. By choosing a set of initial conditions on the periodic solution we are certain that the initial condition is in the basin of attraction of the periodic solution. If the decrease of $V$ is slow enough, the periodic solution will also change slowly, until it suddenly disappears at a certain $v=v_{1}$. $V_{1}$ is the first candidate for $V_{C}$ to be determined in step 3 . It is not recommendable to try to find the periodic solution by starting an integration at $\mathrm{V}<v_{0}$ from a "large" initial condition. The basins of attraction are not known a priori. It is only guaranteed that the transient will end on one of several coexisting stable solutions, if the initial condition for the transient is "sufficiently close" to that solution. When $V>V_{0}$ we expect to find only one stable solution - the periodic oscillation. In some publications this method is called "ramping".

The method for finding the critical speed described above is based on sound mathematical principles. Therefore the resulting $\mathrm{V}_{\mathrm{C}}$ safely can be denoted the critical speed for the vehicle model. Whether it can be denoted the critical speed of the real vehicle is a question of first of all how well all dynamical aspects of the motion of the real vehicle with its real boundary conditions are reflected in the numerical model, and second, how good the numerical solver is. Many of the multibody simulation programs available today will perform satisfactorily.

The parameter variation presents the most serious problem. Much is known about the dependence of the linear critical speed $v_{0}$ on the various parameters, but in contrast little is known about the influence of the same parameters on the critical speed $V_{c^{*}}$. Some tendencies can be found in True and Jensen $(1993 \mathrm{c})$. It is interesting to notice that the influence of a parameter variation may have opposite effects on $V_{C}$ and $V_{0}$. All the critical speeds in True and Jensen (1993c) have been computed for one specific parameter combination at a time using the method outlined above.

\section{AN EXAMPLE}

We examine the Cooperrider model in True (1993a) of a bogie running with constant speed $\mathrm{V}$ on a perfect, stiff, level and straight track. The displacements are measured relative to a coordinate system moving along the straight track with the constant speed of the vehicle. In this moving reference frame the displacements are assumed small. The wheels, axles and the bogie frame are stiff, and friction is only included in the wheel-rail forces.

As wheel and rail profiles we use DSB 82-1 and UIC60 with gauge $1435 \mathrm{~mm}$ and a cant of $1 / 40$.

We solve the system of equations for various values of the speed $V$ in the following way.

First W. Kik's RSGEO program is used to determine the contact points as a function of the lateral displacement. Then the system of nonlinear ordinary differential equations is solved numerically using ISODA. We have used the Vermeulen-Johnson approximation for the creepage-creep force relation. We present three results from True and Jensen (1993c). In the first case the coefficient of adhesion is 0.15 and in the second case the coefficient of adhesion is 0.30 . The wheel base in both 
cases is $2.15 \mathrm{~m}$. In the third case the whee 1 base is $3.2 \mathrm{~m}$ and the coefficient of adhesion is 0.15. True and Jensen found the following values for the linear critical speed and the nonlinear critical speed.

$\begin{array}{ccc} & \begin{array}{c}\text { Nonlinear critical } \\ \text { speed mls/h }\end{array} & \begin{array}{c}\text { Linear critical } \\ \text { speed mls/h }\end{array} \\ \text { Case 1 } & 94.5 & 109.1 \\ \text { Case 2 } & 87.8 & 107.8 \\ \text { Case 3 } & 106.4 & 123.1\end{array}$

The smallest difference between the two critical speeds in this example is $14.6 \mathrm{mls} / \mathrm{h}$ in case 1 and the largest is $20.0 \mathrm{mls} / \mathrm{h}$ in case 2 .

\section{CONCLUSION}

The concept "critical speed" for the onset of hunting of a railroad vehicle is criticized. A unique critical speed is difficult to measure, and the measured critical speed rarely agrees with the one found by the conventional eigenvalue analysis of the linearized mathematical problem. We therefore suggest that another critical speed, $v_{C}$, shall be used in future by the railroad industry as a criterion of acceptance for railroad vehicles. The speed, $v_{c^{\prime}}$ is defined in such a way that it is theoretically guaranteed that no hunting can take place at speeds below $\mathrm{V}_{\mathrm{c}} \cdot \mathrm{V}_{\mathrm{c}}$ can be determined uniquely by numerical methods, and its calculation is no more difficult than doing a simulation. Any good existing simulation program can easily be modified to do the necessary calculations. Since the definition of $v_{c}$ is theoretically justified and its computation is based on proven mathematical principles, $v_{c}$ will definitely be a much better approximation to the true critical speed of the vehicle than the so called critical speed, which is calculated today.

\section{ACKNOWLEDGEMENTS}

This article was conceived after discussions with Alan Carter from British Rail Research and John Elkins from AAR. The author wants to thank both for the inspiration.

The work was supported by the Danish Council for Scientific and Industrial Research under grant no 16-4786.M.

REFERENCES

Stephenson G, Observations on Edge and Tram Railways, 19th March 1821.

Huilgol RR, Hopf-Friedrichs bifurcation and the hunting of a railway axle, Quart $J$ Appl Math 36, 1978, 85-94.

Moelle D and Gasch R, Nonlinear bogie hunting, in Proc. 7th IAVSD Symp. Cambridge, Swets \& Zeitlinger, Lisse, 1982, 455-467.

True $\mathrm{H}$ and Jensen $\mathrm{JC}$, Chaos and asymmetry in railway vehicle dynamics, in Proc. 13th IAVSD Symp. Chengdu, 1993c. In print.

Gasch R, Moelle D and Knothe $K$, The effect of nonlinearities on the Limit Cycles of railway vehicles, in Proc. 8th IAVSD symposium on vehicle systems Dynamics in The Dynamics of Vehicles on Roads and Tracks, JK Hedrick (ed), Swets \& Zeitlinger, Amsterdam/Lisse, 1984, 207-224.

Jaschinski $A$, On the application of similarity laws to a scaled railway bogie model, Doctoral thesis, Technische Universiteit Delft, The Netherlands, 1990.

Knudsen $C$, Feldberg $R$, and Jaschinski A, Nonlinear dynamic phenomena in the behaviour of a railway wheelset model, Nonlinear Dynamics 2, 1991, 389-404. True, $\mathrm{H}$ and Kaas-Petersen, C, A bifurcation analysis of nonlinear oscillations in railway vehicles, in Proc. 8th IAvSD Symposium on Vehicle systems Dynamics in The Dynamics of Vehicles on Roads and Tracks, JK Hedrick (ed), Swets \& zeitlinger, Amsterdam/Lisse, 1984, 655-665.

Kaas-Petersen $\mathrm{C}$ and True $\mathrm{H}$, Periodic, biperiodic and chaotic dynamical behaviour of railway vehicles, in Proc. 9th IAVSD Symposium on Vehicle systems Dynamics in The Dynamics of Vehicles on Roads and Tracks, O Nordstrøm (ed), Swets \& Zeitlinger, Amsterdam/Lisse, 1986, 208-221. 
True, $H$, Some recent developments in nonlinear railway vehicle dynamics, lst European Nonlinear Oscillations Conference, in Proc. of the Intern. Conf. in Hamburg, Aug. 16-20, 1993. E Kreuzer and G Schmidt (eds), Akademie Verlag, 1993a, 129-148.

True, H, Dynamics of a rolling wheelset, Appl. Mech. Rev. Vol. 46, No. 7, part of Chaos and noise in dynamic systems, T Kapitaniak and $\mathrm{J}$ Brindley (eds), 1993b, 438-444 